Murmurations:

Journal of

Transformative

Systemic

Practice
RESEARCH NOTES

\section{Invitations to mutual learning: Reflections on co-researching a social prescribing model of care during the COVID 19 pandemic}

\author{
Leah Salter
}

\section{Volume 3 \\ Issue 2 \\ Spring 2021 \\ Keywords: \\ social prescribing, \\ participant \\ inclusion, \\ mutual learning \\ Covid-19, \\ research process, \\ practitioner \\ research, \\ systemic inquiry}

Abstract

Citation Link
This paper offers some reflections on the creative and ethical approaches to participant inclusion in a research project which is currently taking place in the time of the COVID 19 pandemic. The project is based in the county of Bridgend, South Wales, UK. An account of one of the storytelling projects this paper refers to was published in the last edition of Murmurations (Salter, 2020).

These reflections focus on the early invitation to intervention phase of the research that might traditionally be thought of as the recruitment phase, followed by interview-conversations with participants. The language of recruitment and interviewing suggests or assumes that the researcher goes in blindly and coldly with some kind of promotional campaign to find people they previously did not know and then co-opt them into a study where they are the participant or the object of study. In relational, practice-based research such as the one featured here, this does not reflect the ethics of systemic values nor community-based practice. This paper describes an alternative model to think about co-inquiry as an invitation to mutual learning. The method and the ethics of inquiry are spotlighted in this paper, within an overview of the context to inquiry - that of researching a social prescribing model of care. The paper also offers a brief reflection on learning in this early stage with contributions from coresearchers.

\section{Introduction}

My colleague and I make up a small systemic family and community service in a health board in South Wales. The relational practices we describe offer examples of practice-based research where collaboration with members of the community is centralised. We regard colleagues and community members as co-researchers. We are not separate to community, we are community. This paper reflects multiple conversations and relationships that continue to influence our systemic practices. We view research as an opportunity to engage in mutual learning, a space where learning is shared 
and meaning making is relationally framed through conversation and interaction with other living systems. Nora Bateson (2016) refers to this process as "symmathesy" or "simultaneous learning" that is "necessary for life" (Bateson, 2016).

For our small team, this research is an extension of the practices we are involved in as family therapists, as team consultants, as trainers, supervisors, community workers/ social pedagogy practitioners. We do not view the learning that comes from researching into our practice as separate to the learning we experience when we meet with families, teams, community groups as part of our therapeutic practices. Sheila McNamee invites practitioners to view systemic research as "social intervention" (McNamee, 1988) and we consider we have taken up that invitation. McNamee also invites us to consider the difference between "truth and utility" (McNamee, 1988). In our everyday practices, as researchers and as practitioners, we are not seeking to find any one truth but to engage in processes that have mutual utility. As an aside, it will be useful to discuss if a distinction between practice and research is necessary within "systemic ways of living" (Simon and Salter, 2019) but this is beyond the scope of this paper.

\section{Working with families and communities}

Our therapy work with families in a community mental health context has been disrupted by the social restrictions associated with COVID 19 and we have needed to adapt quickly within this context to position ourselves as being useful to/for our community. We are no longer able to meet with families in person in a clinic setting. Some of those meetings have moved online, where technology allows, and where it was assessed as safe enough. We have also extended our work in the wider community to use our relational skills to support communities to learn and heal, using a whole systems approach to recovery and "storytelling for social change" (Stroh, 2015). This includes online storytelling and creative writing groups for women using mental health services. It also includes a storytelling project called "not to be forgotten stories" (see Salter, 2020) where people who self-identified as being adversely impacted by the pandemic - including adults with mental health diagnoses, NHS (National Health service) staff and members of the community - joined us in an open field in the summer of 2020 to share stories of the pandemic, including how it has affected their wellbeing. The coresearchers/story tellers met with a participatory artist and they worked together to create a tree made of wire and other materials, weaving in key messages from all the stories. Researching the impact of this intervention and other projects we are involved in, including a letter writing project called "voices of experience"- where people share their stories of overcoming challenges with other members of the community in an intergenerational skill share - is one of the sites of this research. The other site of research is a local field or Nightingale hospital (Ysbyty Yr Seren) where patients are recovering from COVID 19. We have an existing connection with the hospital as we offer consultation to the wellbeing team who offer interventions that come under the umbrella of a "social prescribing model of care". Ysbyty, by the way, is the Welsh word for hospital. Seren is the Welsh word for star.

\section{Research in a time of pandemic}

COVID 19 has had a profound impact on our communities, including the mental health and wellbeing of people who have lost someone and those who have recovered from the virus, their families and networks. The pandemic has also adversely impacted people with existing mental health conditions 
and other vulnerable groups. The research that has been most evident in the media has often focused on quantitative data such as mortality and physical recovery rates. Whilst this is important data to be shared and is often a stark reminder of the brutal impact of this virus, it does not show us the detail and depth of how people and communities have been affected. Our developing research aims to address the gap in understanding the lived experience of people in our communities including those who have been adversely impacted in terms of mental health and those hospitalised, by inviting people to share their experiences with us.

\section{A relational ethic of care}

As researchers, just as we are as practitioners, we are led by an ethic of care. An ethic of care is "rooted in receptivity, relatedness and responsiveness" (Noddings, 1984, p. 2). Carolyn Ellis suggests that relational ethics requires researchers to "act from our hearts and minds" and she highlights that research that is relationally attuned and ethics led needs to recognise that our relationships with "participants" changes over time. This means that some of the ethical dilemmas that arise are not directly addressed by ethics committees or research boards (Ellis, 2007). These dilemmas need to be addressed from within the relationship, within the communities they interface with and with responsiveness over time.

Ellis refers to research where the researcher "becomes friendly with" or "gets close" with a community/ population group in order to "find stuff out" and then move back out of the community before writing up their "findings" (Ellis, 2007). This is a model of research that might be more familiar to academics working in research departments who are invited in from outside to do research. My colleague and I both work and live in the same community as our co-researchers. We are invested in the community in a different kind of way and we also need to be transparent about being "insider" or practice-based researchers with existing relationships with many of the people we talk with in this capacity (Salter, 2015, 2017).

We are also working within a stance of openness, of preparedness to learn, of curiosity about how we and others see things (Cecchin, 1987), alongside a preparedness to assume positions of solidarity and justice-doing in community work (Reynolds, 2010, 2013; Salter, 2017, 2018). We are not seeking to create separation between ourselves and the communities we work with/ inquire with. Our curiosity is often sparked by common experiences, including those of injustice. We are not neutral, we do not claim to be neutral in our work and research. Instead we put our professional and social know-how to work in working out with our co-researchers how best to position ourselves. This has the impact of increasing trust in research and professional relationships and generating deeper understanding.

\section{Purposes of the research}

- To contribute to an understanding of people's lived experience of COVID 19 in our community

- To listen and respond to people within our community so they feel their experiences are validated

- To find ways to share this learning with the teams involved in their care so creating a 
direct feedback loop which can carry on the work

- To find ways, with the storyteller, to contribute to a culture of learning and share feedback with the wider community

- To gain an understanding of the difference that a social prescribing intervention might make to personal recovery from those hospitalised with COVID 19

- To develop better understanding of the difference a social prescribing intervention might make to community recovery from the wider social impact of COVID 19

- To contribute to a wider body of knowledge in relation to a social prescribing model of care

- To contribute to a wider body of knowledge in relation to individual and community recovery following COVID 19.

\section{Conversational inquiry}

The method of inquiry we are engaged in could be described as interview based, using storytelling methodology; within a frame of narrative inquiry (Clandinin and Connelly, 2000) and one of community learning (Senge, 1997; Salter, 2020). The word "interview" does not do this relational experience justice though. Interview has limitations in a research context in the way it does in a family therapy context. It suggests that conversation is unidirectional and that only one party has the power to ask questions, whilst the other is obliged to answer. I have previously used the term "conversational inquiry" (Salter, 2018) within a research context and whilst this may also have limitations it aims to offer a thicker description of the process of talking with and learning with. This type of inquiry is one of "co-construction" (Simon and Salter, 2019, 2020; Tomm, 1999) and is relationally framed. The research is not aiming to gather facts or find truths as if they pre-exist out there but is engaged in an evolving process of joint discovery, of mutual learning and transformation.

\section{Co-inquiry}

We are at the beginning of the conversational inquiry - starting to have conversations with people in two different settings. One is with people who have been or are still recovering from COVID-19 in a Nightingale (COVID-19 field) Hospital and have experienced interventions that come under the umbrella of social prescribing model. The other is with people in the community who have already engaged in community wellbeing projects with us. As such the one group are already known to us and we will be revisiting their experience with them and the other group are not known to us but have built a relationship with a member of the hospital wellbeing team who then invites them to share their experience with us. The relational process is important and needs to be attended to. It matters who makes the invitation, and how. From the offset this is viewed as a conversation and choice is in-built. It is made very clear that their choice will not, in any way, effect their health or social care. The conversation might start a bit like this...

"Hey Cerys, you remember the project we all took part in where we worked with the artist 
in the field. My colleague and I are really interested in how people experienced that and how people experienced other projects like that. We are so interested, in fact, that we have decided to put time aside to sit down and talk with all the people who took part. We are also working with some academic researchers and we will be writing up what we have learnt.

How would you feel about sharing your experience with us? It can be a conversation like we are in now, or we could make something together, like a picture, or a poem or a story. You might want to talk with me, or with my colleague who you don't know so well. You can choose which feels better for you. We can do this in whatever way helps you feel comfortable and best captures your story. When we have had our conversation or you have written down your story, you can make as many changes to it as you like.

It's still your story. If you feel like it would be good to share your story with other people, like people involved in your care, or maybe with people who make decisions about what kind of care people receive in this community, we can have a think together about who that might be. If you decide to do this, I will ask you to sign a consent form so that we know it is ok to share your story with other people. This might be in direct ways, like talking with your care co-ordinator (in which case we can name you, if you want us to) or it might be in more general ways, like presenting what we have learnt in conferences and in journals.

We won't use your name or any information that identifies you in that case, so people won't connect your story with you, but they will get to hear it and maybe learn from it. We are doing this because we hope others WILL learn from your experience and maybe this will help change how we do things. We will be talking with other people too and we are curious about whether there are things we can learn by looking at all the different stories together.

But we will also keep your story whole, to learn directly from what you tell us. Does this feel useful for you? It's ok if it doesn't. It's also ok if you don't have the time or the headspace for this right now. This won't stop you being invited to take part in other projects like this one."

This is typical of how some of the conversations begin and as you can see this is an invitation, through an existing relationship. It is not a cold call. People are asked to tell their story about their experience and to share it with others - if they choose. The story can be told in the way that best fits the individual. It might be purely conversational or it might include writing in poetry or prose or might involve a visual representation. The choice is important and seeks to fit with the personalised care that has already been received.

Once all of the conversations have taken place we will then use a narrative inquiry model of "analysis" (Clandinin and Connelly 2000). Narrative inquiry is responsive to time, place and context and holds information as temporary and evolving. As such, the stories are held as the "primary data" or "material" (I prefer the word material) but are understood within the context of constantly evolving relationships and community culture. The stories from this research will not be coded or dissected as if they were static and cold; nor will we separate them from relational processes and other contexts of their production (Simon 2018). They will be maintained as whole, warm and living stories. As 
systemic practitioners we understand the importance of relationships and co-constructed spaces where we collectively create the conditions where people can share stories that have meaning in their lives and create meaning in their lives. We are not seeking to support people to report facts that can be neatly reproduced and we are not seeking to reduce multiple individual stories into one data set/ homogenise stories into one voice. We are though interested in words or phrases that are repeated within or across texts. We think this may teach us about shared experiences because (as systemic practitioners) we consider there is value in the "combination of data" that helps us to hold multiple contexts in mind (Bateson, 2016). Words will be taken from field texts (for verbal stories) and directly from prose from the written stories.

This feedback from a young woman, Jemma, who I have previously co-authored a paper with (Salter and Newkirk, 2019) shows how using poetry as a way of expressing her experiences of social isolation has helped her. "I felt I could be really honest with my words and poetry... I didn't have to tiptoe around topics. I felt people wanted to hear my opinions on things and valued what I said, which was surprising to me."

Jemma was also involved in the "not to be forgotten stories project". She told me that this project meant a lot to her. "The world has felt so chaotic and uncertain... Because there has been so much heartache and anxiety pretty much all around, it has been hard to find the importance in my own part of the story... I could feel myself drowning... Having the opportunity to be heard made me realise how much my story did matter, that all our stories matter. It's easy to feel guilty for struggling when everyone is struggling to some extent, but it is a freeing experience to be able to unload some of it to have permission to."

Another woman, Catrin, who has been working with a writing for wellbeing practitioner, commented, "I've felt more like I can express myself through words... It allows me to really dig deeper."

This early feedback suggests that working with creative practitioners, traditionally outside of health and social care services, has been received positively and has enabled people to feel heard. It seems like these practices have created a sense of space, of depth and breadth. The emphasis on words as creative expression has helped people feel that their words hold value to others. By inviting people to share these experiences with us, and by creating the relational presence for stories not simply to be told, but heard and taken seriously, we hope to enhance that sense of value further as their stories are witnessed in multiple, meaningful ways.

\section{Mutual learning}

By co-inquiring into the impact of a social prescribing approach to community recovery, the research relationally transforms both the teller and the listener of the stories told (Salter, 2018; Salter and Newkirk, 2019; Etherington, 2004). It also directly contributes to a learning culture where NHS staff and members of the community are learning from each other so having a further positive effect on patient, public and staff wellbeing (Salter, 2020).

This model of mutual learning and co-research expands our (where our includes researchers' and coresearchers') understanding of community recovery and continues a learning culture by not just asking questions about this experience but by being in the experience. We consciously aim to avoid taking an outsider "aboutness" position of researching and rather aim for alongside and from within 
collaborative forms of learning (Shotter, 1999, 2005).

Peter Senge (1997) reminds us that there is a significant difference between a teaching hospital, where medical students work alongside qualified medics, and a learning hospital, which is built on a culture of mutual learning. The hospital we are working with, led by a clinical lead with a passion for social prescribing is piloting this second description. Third sector mental health and wellbeing practitioners, storytellers and creative writers, visual artists, alternative therapy practitioners, yoga, dance and movement practitioners as well as community navigators, community coordinators and hospital chaplaincy together make up a multi-skilled wellbeing team that is integrated with the clinical team to construct a holistic package of treatment. We offer a consultative space for these practitioners in which they and we can learn from their experiences, together, as a mutual inquiry.

A clinical lead told me a story where a woman in her 90's who has complex health needs and has been hospitalised many times, said to her "I have never felt so cared for. Everyone knows my name and knows $m e^{\prime \prime}$. Her bedside notes hold all the necessary clinical information to ensure she receives the right medical care to recover from the virus and to attend to her other medical needs. The notes also include information about what she likes to eat, her hobbies and interests, her likes and dislikes, her links in the community, her previous occupation etc. The clinical team and the wellbeing team (NHS staff, independent wellbeing practitioners and third sector colleagues) can access these notes and tailor interventions to support her. This might include offering a session of poetry reciting or creative writing. It might be someone knitting with her, or reading to her, or taking her for a walk through the art trail. This is, in many ways, practice-based research in action. Practitioners, as part of their everyday practice, have taken the time to inquire into this woman's lived experience of being cared for and her lived experience of life beyond the hospital setting. Their findings have fed directly back into her care to assist her in having a better experience. It is an example of curiosity engendering an ethics led, personalised package of care.

If we translate this across the wider field of health care, we propose it is possible to develop and promote a learning environment in which we are all active learners in our practices. As such we remain open to receiving information from the multiple feedback loops that are generated by a relational, responsive and receptive model of research and/as daily practice (St George, Wulff and Tomm, 2015). We are not merely interested then in what differences particular interventions make to the individual, but also what difference this may make to communities.

\section{A note on social prescribing in Wales}

We are invested in developing our understanding of how people in our community have been affected by the pandemic and how their wellbeing and/or recovery may have been impacted by a social prescribing model of care for those who have experienced it. This is a model that has a relatively strong research base in a primary care setting (more so in England than in Wales) but little research base within a clinical or hospital setting or at a secondary care community level. There is "no one set model for social prescribing as it is meant to be responsive to the local needs of the community and use of local resources" (Royal College of Nursing, 2020). However growing evidence suggests that social prescribing can lead to improved health and wellbeing outcomes (Royal College of Nursing, 2020). It is usually aimed at people who are socially isolated and who have long term physical and/or mental health conditions (British Medical Journal, 2019). In essence the aim of this model is to link traditional 
clinical practice with social activities and support systems in the interconnected fields of physical and mental health. This includes social connectivity, engaging with nature, cultural and/or arts-based activities to aid wellbeing and therefore recovery. This model has developed differently in Wales than neighbouring England. In Wales, social prescribing has developed from third sector and community enterprises, making for a diverse range of interventions and differing routes to interventions which are less likely to come from GP referrals and more likely to come from community connectors or social navigators working within the local authority, social housing or in the voluntary sector services. The interventions are likely to have developed from an understanding of local need and may not be networked to wider social interventions. In addition, the people prescribing the intervention may also be delivering them. This is the case for the projects highlighted here, but this is not always the case. One of the challenges of this diverse range of interventions and referral or signposting systems in Wales is being able to track, evaluate and monitor the interventions. As such there is a lack of systematic reviews with associated risks in terms of sustainability.

The therapeutic community projects that we have developed in our small systemic family and community service have grown in much the same way - from practice and from understanding of local need, through local relationships. We have promoted some of the projects using social media and we have delivered the services utilising local resources and networks. This has included a small portfolio of projects using a storytelling and creative arts approach to support wellbeing (Salter and Newkirk, 2019; Salter, 2020). As such we are interested to know more about the usefulness of such a model but under this wider umbrella of social prescribing/ social intervention. The British Medical Journal (2019) suggests that further research is required to build a robust evidence base in social prescribing. We aim to contribute to this base, by learning from the people with the most experience or with most to teach us. Dan Wulff and Sally St George suggest that "the idea of research is within practice". We agree with this and we agree that seeing research as "daily practice" results in methodologies that fit the context and are relationally and ethically driven (Wulff and St George, 2020).

\section{Summary}

This brief paper has highlighted how it is possible to enter into "research as everyday practice" whilst continuing to be ethics lead, relational, responsive and receptive within our practice (Wulff and St George, 2020). We have been researching the benefits of a social prescribing model of care in community settings and at a hospital site. Early feedback suggests that both the interventions and the method of inquiry into the utility of the intervention can support people in our communities to feel heard and validated. Positioning ourselves as co-researchers with members of our practice community enables us to maintain curiosity and openness within a spirit of mutual learning. This can contribute to meaningful dialogue between people receiving care and those involved in their care (directly and indirectly) and can affect system change through effective feedback loops. Inquiring into our own practices as well as being curious about other practices in our communities offers multiple opportunities for mutual learning. This paper uses an example of current research into a social prescribing model of care as a practice example but the points of learning can be generalised across wider systemic practices where research is viewed as a social intervention and a site for mutual inquiry. 


\section{References}

Bateson, Nora (2016). Small arcs of larger circles: Framing through other patterns. Axminster: Triarchy Press.

British Medical Journal (2019). BMJ 2019, 364. https://doi.org/10.1136/bmj.I1285

Cecchin, Gianfranco (1987). "Hypothesising, circularity and neutrality revisited: an invitation to curiosity". Family Process, 26, 405-413. https://doi.org/10.1007/978-3-319-15877-8_930-1

Clandinin, D. Jean and Connelly, F. Michael (2000). Narrative Inquiry. San Francisco, CA: Wiley

Ellis, Carolyn (2007). "Telling secrets, revealing lives: Relational ethics in qualitative research with intimate others." Qualitative Inquiry. 13(1), 3-29. https://doi.org/10.1177/1077800406294947

Etherington, Kim (2004). Becoming a reflexive researcher. London: Jessica Kingsley.

St George, Sally; Wulff, Dan and Tomm, Karl (2015). "Research as daily practice: Introduction to the special section". Journal of Systemic Therapies. 34(2), 1-2. https://doi.org/10.1521/jsyt.2015.34.2.1

McNamee, Sheila (1988.) “Accepting Research as Social Intervention: Implications of a Systemic Epistemology". Communication Quarterly 36(1), 50-68. https://doi.org/10.1080/01463378809369707

Noddings, Nel (1984). Caring, a feminine approach to ethics \& moral education. Berkeley: University of California Press.

Reynolds, Vikki (2010). "Doing justice as a path to sustainability in community work". https://bit.ly/2TeWiEQ Reynolds, Vikki (2013). "“Leaning in” as imperfect allies in community work”. Narrative and conflict. Explorations in Theory and Practice. 1(1), 5--75. https://doi.org/10.13021/G8ncetp.v1.1.2013.430

Royal College of Nursing (2020). Social prescribing. https://www.rcn.org.uk/clinical-topics/public-health/selfcare/social-prescribing.

Salter, Leah (2015). "From victimhood to sisterhood - A practice-based reflexive inquiry into narrative informed group work with women who have experienced sexual abuse". European Journal of Psychotherapy and Counselling 17(4), 1-16. https:// 10.1080/13642537.2015.1095215

Salter, Leah (2017). "Research as resistance and solidarity: 'Spinning transformative yarns' - A narrative inquiry with women going on from abuse and oppression." Journal of Family Therapy, 39(3), 285-494.

https://doi.org/10.1111/1467-6427.12172

Salter, Leah (2018). "Acts of resistance: A reflexive inquiry into narrative group work with women who have experienced abuse or oppression". Unpublished doctoral thesis. University of Bedfordshire.

http://hdl.handle.net/10547/623290

Salter, Leah (2020). "Stories 'matter': Storytelling as community learning within a whole systems approach to recovery." Murmurations: Journal of Transformative Systemic Practice, 3, 1, 44-67.

https://doi.org/10.28963/3.1.13

Salter, Leah \& Newkirk, Jemma (2019). "Collective storytelling for health: A three-part story." Storytelling, Self, Society: An Interdisciplinary Journal of Storytelling Studies. 15(1), 108-129.

https://doi.org/10.13110/storselfsoci.15.1.0108

Senge, Peter (1990). The fifth discipline: The art and practice of the learning organisation. London: Random House.

Shotter, John (1999). “Writing from within 'living moments': 'Withness-writing' rather than 'aboutness- 
writing'." Fourth National Writing Across the Curriculum Conference: Multiple Intelligences, Cornell University, June 3-5 1999.

Shotter, John (2005). “Goethe and the refiguring of intellectual inquiry: From 'aboutness'-thinking to 'withness' thinking in everyday life." Janus Head. 8(1), 132-158.

Simon, Gail (2018). “Eight Criteria for Quality in Systemic Practitioner Research". Murmurations: Journal of Transformative Systemic Practice. 1(2), 42-60. https://doi.org/10.28963/1.2.5

Simon, Gail \& Salter, Leah (2019). "Transmaterial Worlding. Beyond Human Systems". Murmurations: Journal of Transformative Systemic Practice. 2(2), 1-17. https://doi.org/10.28963/2.2.2

Simon, Gail \& Salter, Leah (2020). "Transmaterial Worlding as Inquiry". Chapter in The Sage Handbook of Social Construction. Eds. Mary Gergen, Sheila McNamee et al. London: Sage Publications

Stroh, David (2015). Systems thinking for social change. White River Junction, VT: Chelsea Green.

Tomm, Karl (1999). “Co - Constructing Responsibility”. In McNamee, Sheila and Gergen, Kenneth J. (Eds.) Relational Responsibility: Resources for Sustainable Dialogue. Thousand Oaks, CA: Sage.

Wulff, Dan and St George, Sally (2020). "We are all researchers." Chapter in The Sage Handbook of Social Construction. Eds. Mary Gergen, Sheila McNamee et al. London: Sage Publications.

\section{Acknowledgements}

Thanks to those who have contributed/ are contributing to this research project, including: Jemma and Catrin and multiple other co-researchers yet to be named. Thanks also to my colleagues John Doran, Joanne Sullivan, Esyllt George, Liza Thomas-Emrus, Natalie Bell, Juan Delport and colleagues in our Research and Development Department. Wider contributors include Christina Thatcher and Kate North of Cardiff Metropolitan University, Emily Underwood-Lee and Jodie Allinson of University of South Wales / George Ewart Evans Centre for Storytelling, Carolyn Wallace and Megan Elliott of University of South Wales / Wales school for Social Prescribing Research. Thanks also to our local authority colleagues, Andreas Van Wyk and his colleagues at Stronger Together Bridgend and to Uschi Turoczy, writing for wellbeing practitioner and Sue Hunt, visual artist plus the whole wellbeing and clinical teams at Ysbyty'r Seren. Much appreciation and apologies to anyone who is mistakenly left out of this list.

\section{Author}

Leah Salter, DProf, is a systemic psychotherapist and supervisor working in the NHS in Wales within adult mental health services. She has research interests in storytelling, group work with women, research as resistance and solidarity practices. Leah is a doctoral supervisor and tutor at the University of Bedfordshire's Professional Doctorate in Systemic Practice Programme and is Co-Director for the Centre of Systemic Studies. The centre is the new host of The Family Institute Wales. She lives on the coast in South Wales.

Leah Salter, University of Bedfordshire, University Square, Luton, LU1 3JU.

E-mail: leahksalter@gmail.com

URL: https://www.researchgate.net/profile/Leah_Salter 


\section{Citation}

Salter, Leah (2021). Invitations to mutual learning: Reflections on co-researching a social prescribing model of care during the COVID 19 pandemic. Murmurations: Journal of Transformative Systemic Practice, 3(2), 109-119 https://doi.org/10.28963/3.2.8 